\title{
LONG TERM VARIABILITY IN CP STARS ${ }^{1}$
}

\author{
HERMAN HENSBERGE \\ Koninklijke Sterrenwacht van België \\ Ringlaan 3, B-1080 Brussels, Belgium
}

\begin{abstract}
A search for CP2 stars with long photometric periods during more than one decade has led to the detection of the long period variables HD 94660 and HD 116458. Furthermore, in the case of $\gamma \mathrm{Equ}$, the $\mathrm{CP}$ star with the longest magnetic period, a monotonic change of the light output has been measured. Light curves are presented for the known long period variables HD 187474 and HD 188041. The results on the other stars in the programme are shortly discussed.
\end{abstract}

\section{INTRODUCTION}

Magnetic chemically peculiar stars are characterized by relatively small periodic changes in their light output, the character of which might change drastically with wavelength. The same periodicity appears also in magnetic and spectroscopic observations, and is understood in terms of the oblique rotator model. The overwhelming majority of the periods is of the order of 1 - 10 days, however the tail of the period distribution extends to tens of years. In other words, the periods are spread over 5 orders of magnitude.

Table 1 summarizes the periods longer than 100 days mentioned in the catalogue of Catalano and Renson (1984). In the majority of the cases, the original detection was made spectroscopically. This contrasts with the knowledge on periods shorter than 100 days, which is mainly built from photometric observation. Indeed, it is in general easier to derive the periodicity from photometry. In fact, only a small fraction of the periods would be known presently if one would have to omit the detections due to small amplitude light variability. This implies, assuming as a working hypothesis that the long period stars might have similar observational characteristics, that a considerable fraction of them could still be undetected. The only way to avoid such a conclusion, would be to assume that all long period stars are prominent spectrum, light or magnetic variables.

Thus, the frequency of the stars with longer periods is presently badly known. The observed frequency of $3.5 \%$ sets only the lower limit. Hensberge et al. (1984) argue that the upper limit may be set at $13 \%$. Progress may only be

\footnotetext{
${ }^{1}$ Based on observations collected at the European Southern Observatory in the framework of the project on Long Term Photometry of Variables.
} 
Table 1: Long period CP stars from Catalano and Renson (1984). The listed periods are up-to-date values.

\begin{tabular}{|c|c|c|}
\hline star & $\begin{array}{l}\text { period } \\
\text { (days) }\end{array}$ & original detection \\
\hline HD $2453=$ GR And & 525: & light, Wolff (1975) \\
\hline HD $9996=$ HR 465 & 8400: & spectrum, Preston and Wolff (1970) \\
\hline HD $110066=$ HR 4816 & $4900 ?$ & light, Wolff (1975) \\
\hline HD $126515=$ FF Vir & 130 & magnetic, Preston (1970) \\
\hline HD $187474=$ HR 7552 & 2345 & magnetic, Babcock (see Mathys,1991) \\
\hline HD $188041=$ HR 7575 & 224 & magnetic, Babcock (1953) \\
\hline $\mathrm{HD} 201601=\gamma \mathrm{Equ}$ & $\geq 27000$ & magnetic, Bonsack, Pilachowski (1974) \\
\hline HD $221568=$ Osawa star & 159 & light, Osawa et al. (1965) \\
\hline
\end{tabular}

expected from precise differential photometry over a long time base line. In the next sections, I will describe and summarize such an ongoing observational effort in the southern hemisphere. Through the detection of long period stars, this project aims to contribute to a better empirical definition of the long period tail. Does this tail extend continuously and monotonically, or will it show evidence for a bimodal distribution as could be expected when another mechanism than rotation would be responsible for the longer periodicities? Alternative models have been proposed for particular cases of secular variability, but the correlation of long periods with extremely sharp-lined spectra, and the observed range of the variations that falls inside the domain of the light, spectrum and magnetic variations observed in the stars with shorter period, suggest strongly that it might be unnecessary to invoke another mechanism. However, if all these periods are interpreted as rotational periods, then the main challenge lies in the confrontation of observation with theory: what physical process is responsible for this extreme diversification in rotation periods? Another relevant question is, whether the long period stars are distinct from their short period counterparts in any other characteristic than the period length. Such and other questions can obviously only be tackled when a statistical significant number of long period stars will have been detected and studied with diverse astronomical techniques.

An alternative model discussed recently in the literature, is the model of forced precession of a star gravitationally influenced by a companion, applied to HD $152107=52 \mathrm{Her}$ (see Gerth, 1990 and references therein). The fact that in particular, rare cases, other periodic phenomena than rotation and rapid oscillations might cause measurable contributions to the observational quantities is also pertinent on shorter time scales, see e.g. the occurrence of two periods in the light of HD 25267 (Manfroid, Mathys and Heck, 1985). Characteristic of 


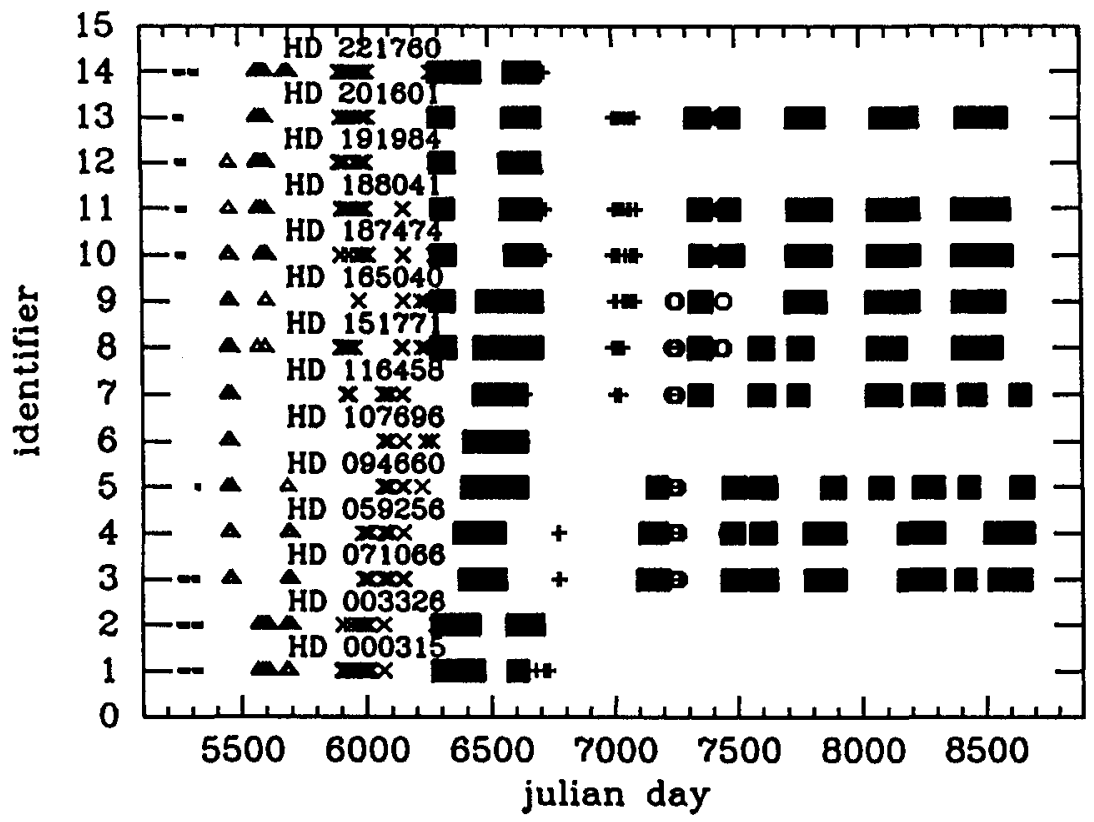

Figure 1: CP2 stars checked for long term variability. Filled squares refer to the Danish $50 \mathrm{~cm}$ telescope, others to instrumental configurations on the ESO $50 \mathrm{~cm}$ and the Bochum $60 \mathrm{~cm}$ telescope, all at La Silla, E.S.O.

all these cases is however that the rotational modulation is measurable together with the other cause.

\section{LONG TERM PHOTOMETRY AT E.S.O. : OBSERVATIONAL ASPECTS}

Early attempts to set up more or less regular photometric observations, in the framework of the European Working Group on Ap Stars, have been described in Hensberge et al. (1984). Since October 1982, a project on Long Term Photometry of Variables was started. It operates under impulse of C. Sterken and J. Manfroid, and coordinates long term efforts at E.S.O. on many kinds of variable objects (see e.g. Sterken, 1983 and Manfroid et al., 1991). Bright southern CP2 stars which were found previously to be photometrically (almost) constant over at least two weeks, and which are not known to show rotational broadened lines, were selected for systematic observation in the Strömgren photometric system, differentially to two comparison stars. Quite unsatisfactorily, for about half of the 14 selected stars, there appeared to be a substantial risk for faster rotation as no information on line width could be found. A posteriori, however, these stars have turned out to be useful in assessing the stability of the instrument as none of the faster rotators shows any evidence for long term variability.

The observations on candidate long period CP2 stars are summarized in 
Figure 1. The last six years, we are in the comfortable situation that the large majority of the observations are performed in the same instrumental system, i.e. as simultaneous uvby photometry at the Danish $50 \mathrm{~cm}$ telescope on La Silla. Initially, the bulk of the observations was obtained in a non-simultaneous mode at the Bochum $60 \mathrm{~cm}$ telescope and the E.S.O. $50 \mathrm{~cm}$ telescope, with the use of different uvby filter sets (up to 1984) and different types of photomultipliers (Manfroid et al., 1991).

The conclusions presented in the next sections will be solely based on the observations carried out at the Danish telescope. Other observations are only referred to insofar as they are useful to extend these conclusions over longer time intervals. The experience with all these different systems convinced me that extreme care should be exercised whenever interpreting data obtained with different instrumentation (see e.g. also Manfroid and Sterken, 1987). The use of different instrumental versions of a photometric system might mimic, with respect to the common amplitudes encountered in CP2 stars, definite variability for a minority of stars, even when using the same filter set but another type of photomultiplier. Although transformation to the standard system does improve the situation (often to an unsatisfactorily extent compared with the accuracy of the data), it does not for all stars. Moreover, transformation to the standard system might introduce false information in the shape of the lightcurves, most pronounced in the case of a prominent wavelength dependence (see the subsection on HD 188041), because the mathematical connections defined in the transformation equations have no relation to the physical processes underlying the wavelength dependency of the light variations. From the experience gathered in this project, I consider conclusions about long term variability of CP2 stars, derived from intercomparison of differential photometry relative to the same comparison star but with a different version of the photometric system as inappropriate, and as indicative only when differences exceed 0.04 mag in $y, b$ or $\mathrm{v}$, or up to $0.08 \mathrm{mag}$ in $\mathrm{u}$.

Details about the observation and reduction procedure will be discussed fully in Hensberge et al. (1993), and are concisely summarized here. The standard observation procedure consists of series of measurements comp.1-CP2comp.2-CP2-comp.2-CP2-comp.1 . All the data obtained in one instrumental system are reduced as one entity by J. Manfroid, the older ones thus being continuously reprocessed for obtaining a homogeneous data set (Manfroid et al., 1991). The internal consistency within each group is checked and used to define error bars (shown in the phase diagrams). My present evaluations indicate that they provide useful lower limits to the external differential errors, although the errors in $u$ tend to be underestimated on the average by $25 \%$ for plausible reasons. The large majority of the differential data have an accuracy better than $2.5 \mathrm{mmag}$ in $\mathrm{y}, \mathrm{b}$ and $\mathrm{v}$, better than $3.5 \mathrm{mmag}$ in $\mathrm{u}$, better than 2 mmag in $\mathrm{v}-\mathrm{b}$ and $b-y$ and better than 3 mmag in $u$. 

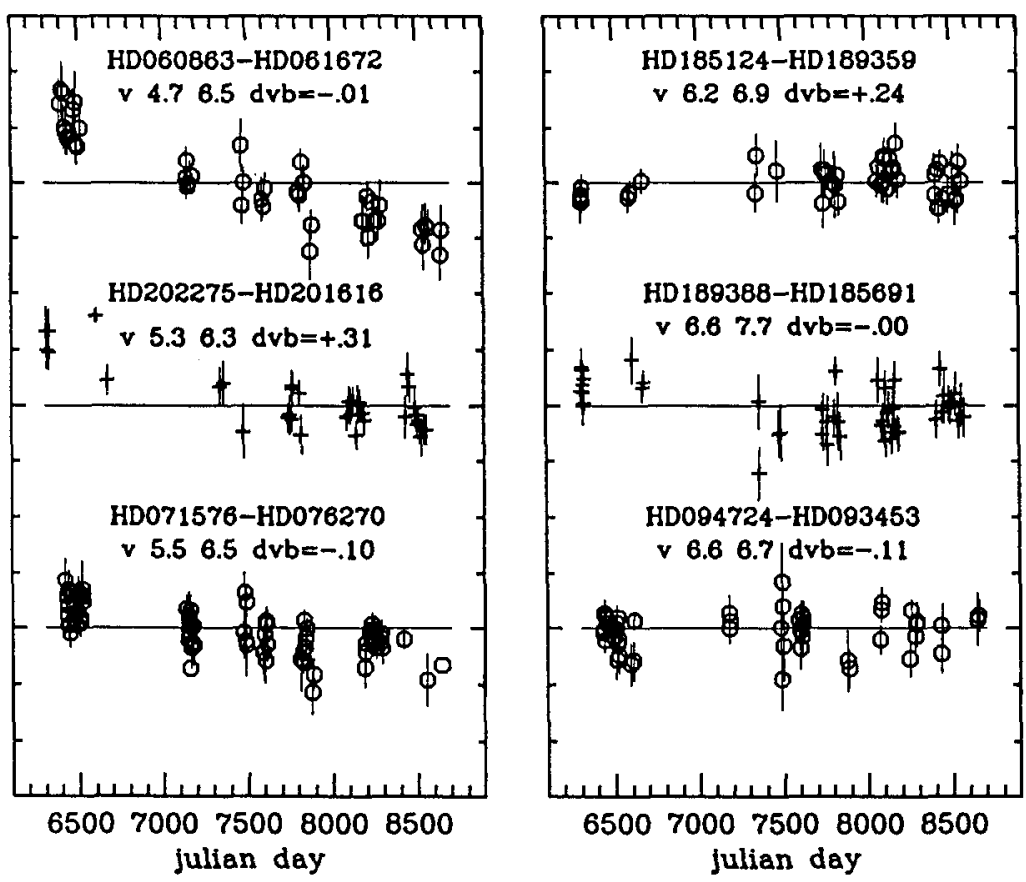

Figure 2: Differential v-b values for couples of comparison stars, all in the sense brighter - fainter star. The brightness in $v$ and in the differential $v-b$ are indicated. Small tickmarks separate $0.005 \mathrm{mag}$ intervals.

\section{INSTRUMENTAL STABILITY}

Checks on the instrumental stability are commonly performed during the general data reduction. Additional tests were performed by evaluation of the differential behaviour of couples of comparison stars, and by comparison of residuals with the estimated internal errors for such couples and for apparently constant programme stars. I will not discuss this matter at length here, since in many aspects the stability is remarkably high. However the instrumental performance in the $\mathrm{v}$ channel shows a gradual trend with time that needs to be commented.

Figures 2 and 3 illustrate that the measured differential value in the $v$ channel depends on the magnitude difference between the stars at a rate that gets more pronounced for the brightest stars. This effect has been isolated only very recently and might be due to a gradual change in the dead time correction to be applied for the $v$ photomultiplier. In all three other channels, the effects are insignificantly small. Once the differential data are corrected for this trend, which amounts to 2 mmag per 1000 days per magnitude for the brightest stars in our programme, then the residuals in the differential $v$ values for comparison stars are indistinguishable from those in $b$ and $y$ and typically only $10 \%$ higher 


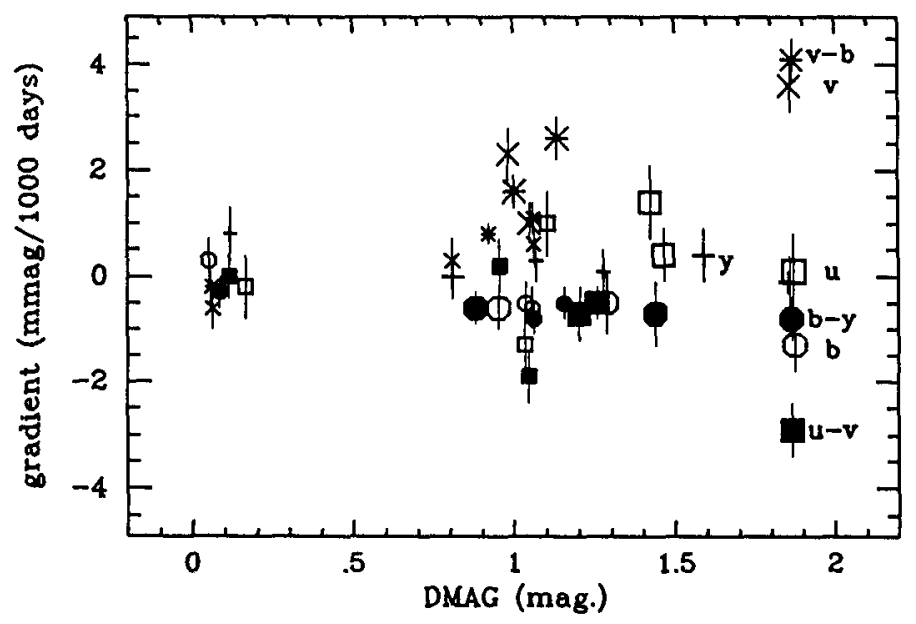

Figure 3: Gradients in differential values for all couples of constant comparison stars measured over more than 2000 days. Larger symbols indicate couples with at least one star brighter than $v=5.5$. Notice the significant trends for the cases that involve the $v$ channel.

than the lower limit set by the internal error estimates.

It is interesting to notice that this correction removes the long term trend apparent in the results for the very bright CP2 star HD 165040, in v more than $1.5 \mathrm{mag}$ brighter than both comparison stars. It shows once more the risk of deriving false conclusions due to unnoticed, quite subtle instrumental effects.

\section{LIGHTCURVES FOR KNOWN LONG PERIOD STARS}

HD $188041=$ HR 7575

This star is included as a performance check on the system used. As a peculiar star, it might be more sensitive to small instrumental changes. It shares with the programme stars the slow photometric variation, which is pronounced in Strömgren $v$ and very subtle in the other channels. It was observed relative to HD 189359 (A2) and HD 185124 = HR 7460 (F3IV). The need for a slight revision towards a smaller value of the period than the one based on Jones and Wolff (1973), expressed by Mathys (1991) based on new magnetic data, is fully confirmed. The period has been fixed at $223.9 \pm 0.2$ days. Figure 4 shows the phase diagrams with phase 0 referring to the minimum of the averaged longitudinal magnetic field at JD 2432323.0. The curves represent fits to the weighted data by a cosine and its first harmonic. The lack of axisymmetry in the $v$ band is evident.

The most obvious difference with the photometry of Jones and Wolff (1973) is the lack of variability in the $u$ channel, which is closer to the result of Musielok et al. (1980) in the Vilnius ten colour photometric system. This does very proba- 

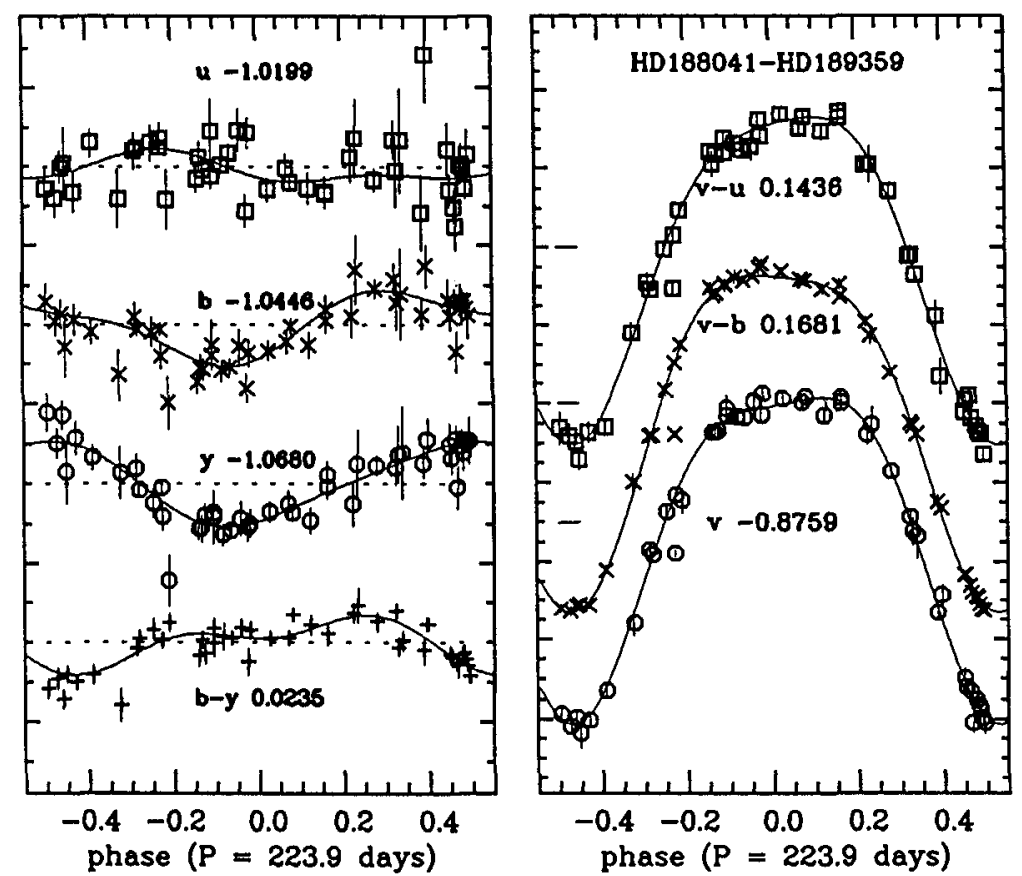

Figure 4: Strömgren photometry of HD 188041 expressed relative to HD 189359. Large tickmarks on the vertical axis separate $0.02 \mathrm{mag}$ intervals.

bly NOT represent a real difference, as we easily obtain in different instrumental systems variations in $u$ in phase or in antiphase with $\mathbf{v}$ after transformation to the standard system. During such a transformation, part of the large variation in the $v$ band is transferred to the $u$ band. Depending on the numerical coefficients, it produces an in-phase or in-antiphase mirror image of the $v$ lightcurve.

HD $187474=$ HR 7552

The long term character of its magnetic variations was detected long ago by Babcock, although apparently never published. The data are now available in Mathys (1991), who updated the period to $2345 \pm 15$ days taking into account his recent measurements. Phase 0 refers to the positive extremum of the averaged longitudinal magnetic field at JD 2446637.039 .

The photometry relative to the stars HD 185691 (A2V) and HD $189388=$ HR 7639 (A2.5V) confirms the period (notice that the most recent observations, at phase 0.78 , almost close the cycle). Near phase 0.95 , in the same rotation cycle, observations were also obtained at the ESO $50 \mathrm{~cm}$ telescope, allowing to tie in observations made in the phase interval $0.10-0.15$ with exactly the same instrumentation. These data were used to constrain better the fits shown in Figure 5. Dashed lines refer to the inclusion of a small term in $3 \phi$ in those 

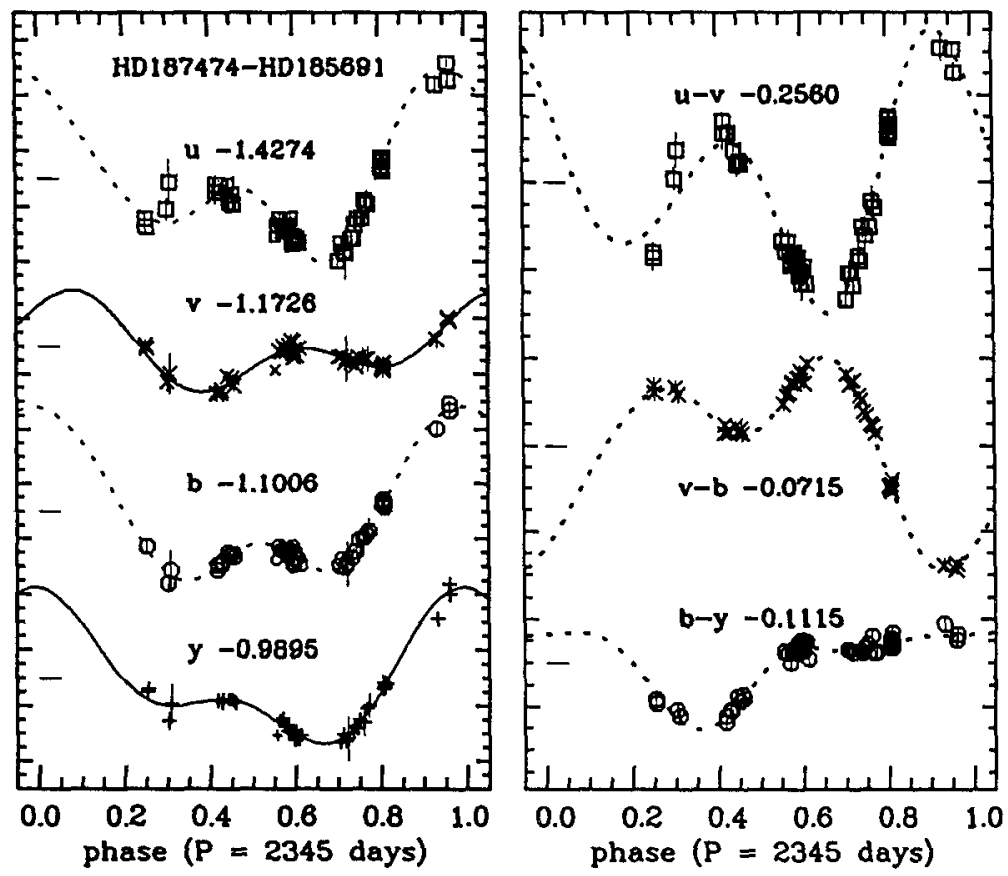

Figure 5: Strömgren photometry of HD 187474 expressed relative to HD 185691. Large tickmarks on the vertical axis separate $0.02 \mathrm{mag}$ intervals.

cases wherein the cosine and its first harmonic reproduced the data somewhat inadequately. The most prominent variability occurs during the one third of the cycle when the metallic spectrum and the $\lambda 5200$ depression are prominent. The magnetic field measurements suggest strongly that the weaker magnetic pole is then close to the line of sight. The colour variations and their phase relation are quite remarkable in this star. A note by Schöneich (1984) based on few ANS ultraviolet observations attracts attention to the large amplitude variability in the ultraviolet, showing a maximum near phase 0.25 .

\section{NEWLY DISCOVERED LONG PERIOD STARS}

HD $94660=$ HR 4263

The strong magnetic field in this star was detected by Borra and Landstreet (1975), and later confirmed by Mathys (1991); no polarity reversal has been observed up to now. Mathys comments that the star shows a very small projected rotational velocity. The apparent photometric constancy over weeks was observed in 1975 and 1977 by Heck and by Manfroid.

The slow light variability of this star was obvious in the very early stages 
of the project, but the use of various instrumental systems lead to suspect a periodicity of the order of hundreds of days. This is one of these few peculiar stars for which the output in the u channel, measured differentially to HD 94724 (A0V) and HD 93453 (A4IV), is very sensitive to the instrumental parameters.
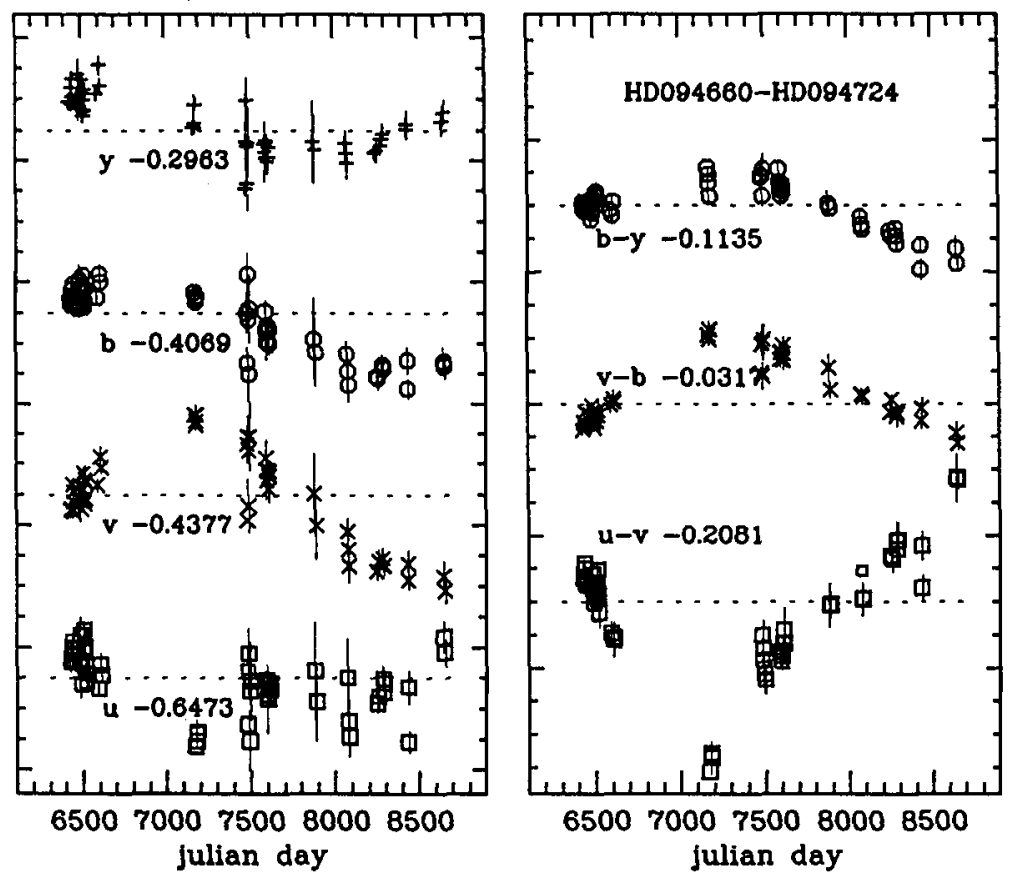

Figure 6: Strömgren photometry of HD 94660 expressed relative to HD 94724. Large tickmarks on the vertical axis separate 0.02 mag intervals.

Figure 6 displays the measurements on the Danish $50 \mathrm{~cm}$ telescope. Observations at the ESO $50 \mathrm{~cm}$ telescope in the period JD 2446000-200 can be tied in except for $u$ (the type of photomultiplier being different from the one used in the later observations at that telescope) and are all near to the same level as the observations near JD 2448650, suggesting that the period might be close to 2700 days. Still, the lightcurve could turn out to have a more complicated character corresponding to a longer period, so this result needs to be confirmed in the next years, when the star is expected to become brighter relatively fast in the $\mathrm{v}$ band if the proposed period is correct.

HD $116458=$ HR 5049

This sharp-lined star is a spectroscopic binary with an orbital period of 126.18 days (Dworetsky, 1982). It is famous for the extreme strength of its cobalt lines. The discovery of its magnetic field goes back to Wood and Campusano (1975). Maitzen and Wood (1977) found the star rather constant in light and 

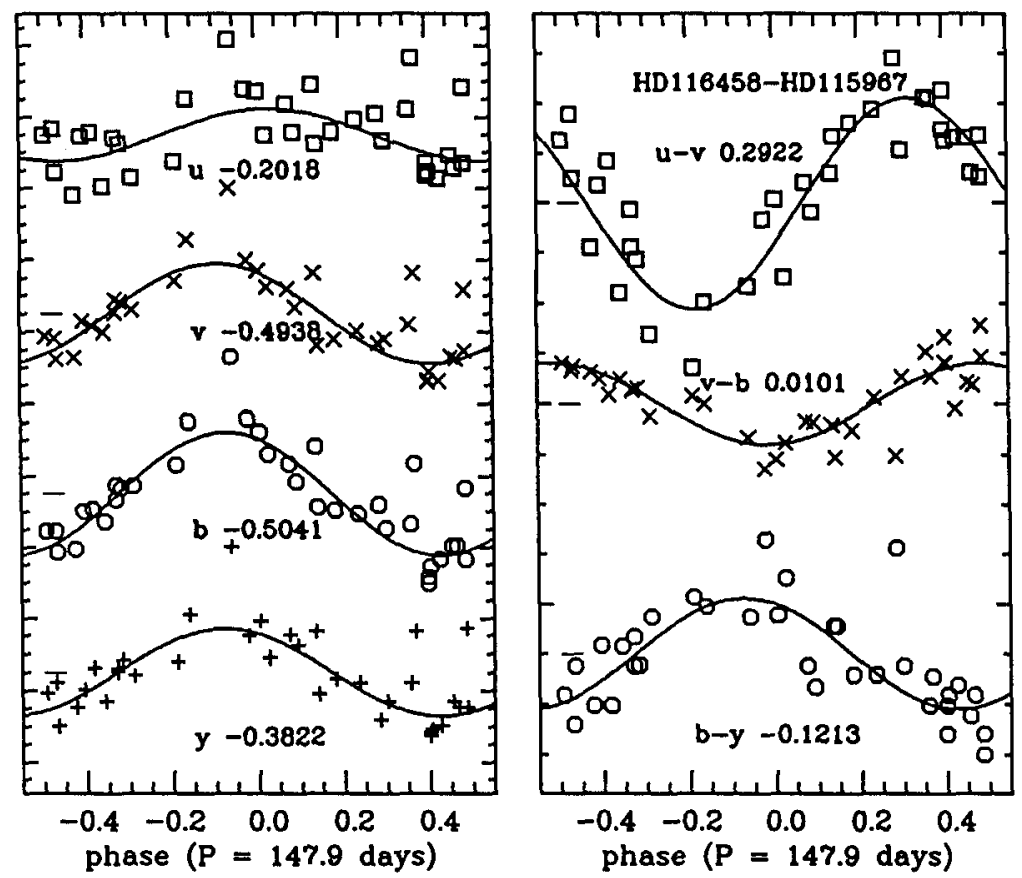

Figure 7: Strömgren photometry of HD 116458 relative to HD 115967. Large tickmarks on the vertical axis separate $0.02 \mathrm{mag}$ intervals.

colour over ten day intervals in February 1973 and March 1974, which was later confirmed by Renson (1977).

The star is measured differentially to HD $115967=\mathrm{HR} 5030(\mathrm{~B} 6 \mathrm{~V})$ and HD $116579=$ HR 5051 (B9V). The latter proved to be slightly variable, so the comparison is made solely to HD 115967. As I did not notice for a long time that the observing sequence was changed to the short mode comp.1-CP2-comp.2, the quality of the data is lower than for the other objects and the internal error analysis cannot be applied. As a consequence, by comparison with the results of the error analysis in other groups, it might be expected that roughly $10 \%$ of the data are of very low quality. In spite of this, the periodicity of the data can be recovered and fixed to $147.9 \pm 0.6$ days (Figure 7). Phase 0 is chosen arbitrarily at JD 2447600.0 . The variations are sinusoidally within the observational accuracy. A weighted fit to the averaged longitudinal magnetic field data in Albrecht et al. (1977), referring to 1970-75, and those of Mathys (1991) in 1986-88 gives

$$
\begin{array}{r}
\left.<H_{z}\right\rangle=-1830-370 \cos (\phi+0.09) \\
\pm 50 \pm 80 \quad \pm 0.03
\end{array}
$$

and supports the photometrically derived period (Figure 8). 


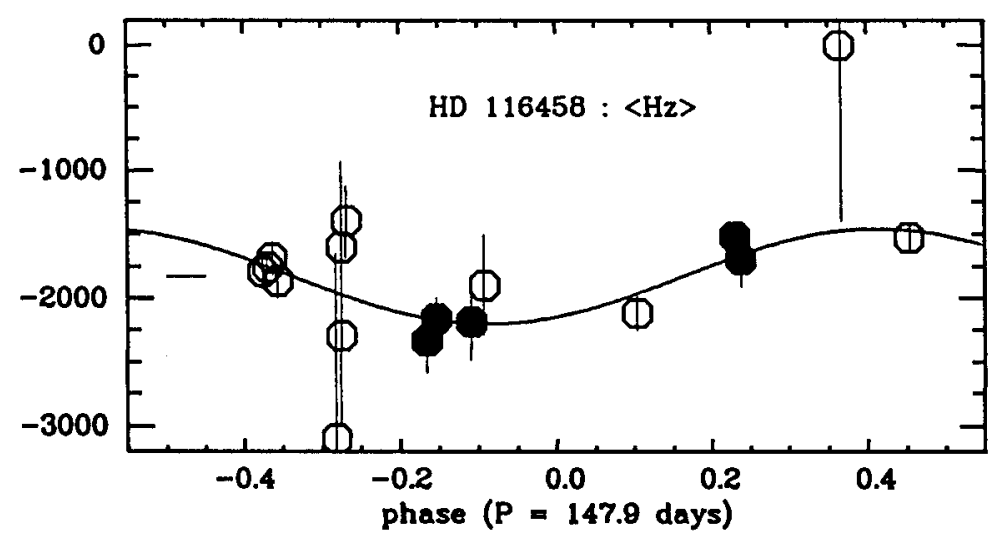

Figure 8: Longitudinal magnetic field data from Albrecht et al. (1977) and Mathys (1991) (resp. open and filled symbols) according to the photometrical ephemeris.

\section{THE LONG TERM TREND IN HD 201601 $=\gamma$ EQU}

This extremely sharp-lined star is famous for the slow gradual change in its averaged longitudinal magnetic field component, well documented by several observers (Babcock, 1958; Bonsack and Pilachowski, 1974; Scholz, 1979; Mathys, 1990). It changed gradually from about $0.6 \mathrm{kG}$ in 1947 to less than $-1 \mathrm{kG}$ presently, changing sign in 1970/71, so that the 72 year cycle suggested by Bonsack and Pilachowski might be still too short. Rotation induced light variability has never been traced satisfactorily, although several suggestions, based on a quite unsatisfactorily amount of data, are in the literature (van Genderen, 1971; Winzer, 1974; Deul and van Genderen, 1983).

The star is measured differentially to HD $201616=\mathrm{HR} 8097$ (A2Va) and HD $202275=$ HR $8123($ F5V +..). The most obvious gradient apparent in Figure 9 is in $v$ and $v-b$ and corresponds, in units of mmag per 1000 days, to $+6 \pm 0.5$ in $v$. The systematic instrumental effect expected in this case is in the opposite direction and much smaller ( roughly -1). Another method of verifying the reality of the observed gradient is to compare the star separately to HD 201616 ( 1 mag fainter in v) and HD 202275 (only 0.1 mag fainter). In addition, the presence of significant gradients in all colours (b-y: $+2 \pm 0.4$; v-b: $+3 \pm 0.5$; u-v: $-3 \pm 0.6$ ) adds evidence to the reality of the observed changes. Ideally, one would like however to have this result confirmed independently. Moreover, it is not at all clear since when such a trend with time might have manifested itself, or will continue. In spite of all caution to be taken when making external comparisons, it is tempting to notice that the colours observed by Wolff and Morrison (1973) in 1963 and 1970-71 do not seem to be significantly different from those obtained recently. At least, the result is encouraging enough to continue the efforts on this star. 

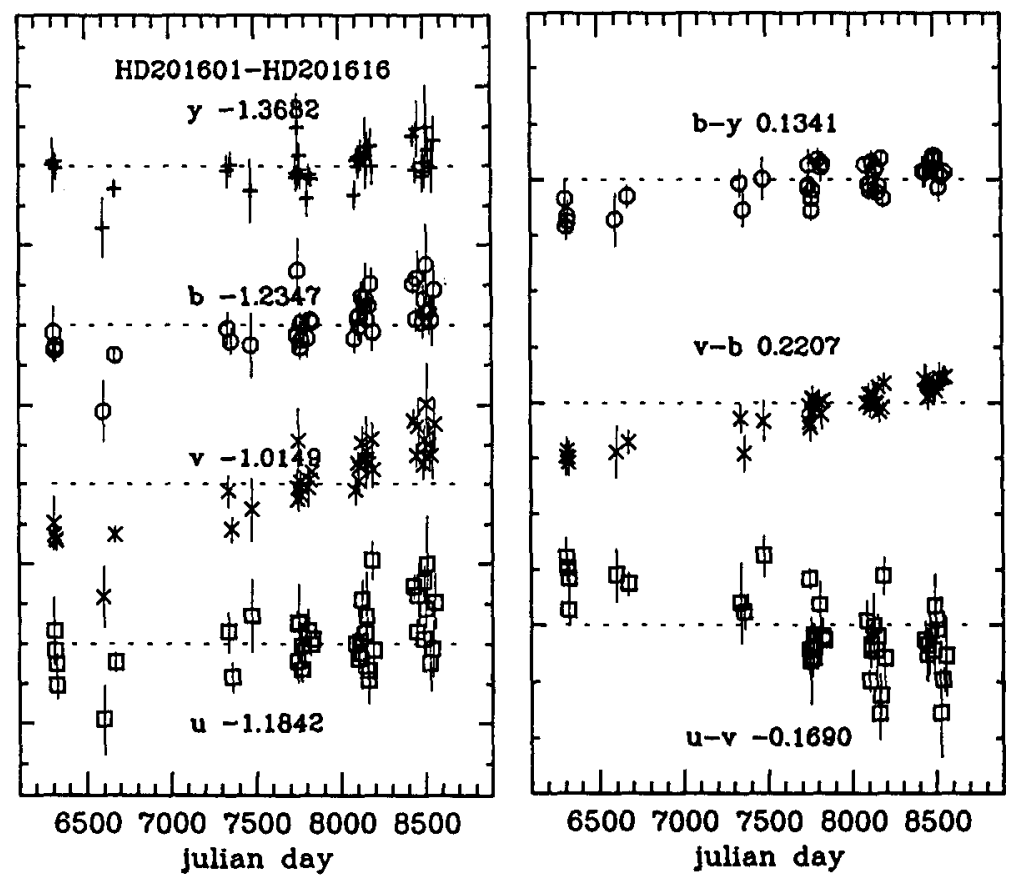

Figure 9: Strömgren photometry of HD 201601 expressed relative to HD 201616. Large tickmarks on the vertical axis separate $0.02 \mathrm{mag}$ intervals.

\section{CP STARS WITHOUT LONG TERM LIGHT CHANGES}

In the course of this project, colleagues informed me that the spectra of HD 3326 $=\mathrm{HR} 151, \mathrm{HD} 59256=\mathrm{HR}$ 2863, HR $191984=\mathrm{HR7717B}$ and HD $221760=$ $\iota \mathrm{Phe}$, and to a lesser degree HD $165040=\pi$ Pav, show rotationally broadened lines. Actually, three of them were already taken off the programme: HD 191984 which showed evidence of slight variability in the u filter (at most $0.01 \mathrm{mag}$ ) over shorter time intervals, and HD 3326 and HD 221760, both of which seem to remain constant in the visible to better than 3 mmag. HD 3326 shows $\delta$ Scuti type variability with an amplitude of $1.8 \mathrm{mmag}$ (Kurtz 1982; Kreidl, 1985). Intensive observations on HD 221760 in October 1982 don't confirm the 12.5 day period suggested by van Genderen (1971). The observations of HD 191984 include the light contribution from its companion at a distance of 3 ". The two other stars were observed until now and the data await detailed analysis, but it is obvious that any variability will not exceed a level of 5 mmag.

HD $71066=$ HR 3302 has according to Mathys very sharp lines, but its period cannot be too long. The star shows repeatedly evidence for variability over a range of at most $0.01 \mathrm{mag}$. Further analysis might well reveal a periodicity in the order of several weeks, but it is certainly not much longer. 


\section{CONCLUSIONS}

More than one decade of long term monitoring of a very limited number of bright apparently photometrically constant CP2 stars has led to the detection of two long period stars and to the observation of monotonic colour and brightness gradients in $\gamma$ Equ. All five stars with long periods are sharp-lined and show Zeeman split lines in high resolution unpolarized light (Didelon, 1987; Mathys, 1990; 1992; Preston, 1971; Scholz, 1979). Some broad-lined stars were included in the programme, but none of them shows any sign of long term variability. The advantages of using one instrumental system throughout, and, even then, the necessity to check continuously the instrumental stability is painfully evident.

\section{ACKNOWLEDGEMENTS}

This work would have been impossible without the dedication of C. Sterken in organizing continuously the preparations for the observing runs and that of $\mathrm{J}$. Manfroid in providing the data reduction support. Numerous observers contributed generously to this project: $O$. Stahl, M. de Groot, D. vander Linden, F.-J. Zickgraf, H. Ott, R. Schulte-Ladbeck, T. Hageman, H. Mandel, H. Duerbeck, F. Decker, H. Cuypers, A. Bruch, A. Reitermann, M. Burger, A. Jorissen, H. Steenman, R. Madejski, A. Figer, R. Duemmler, M.V. Mekkaden, A. Heck, Y.K. Ng, E. Bibo, N. Vogt, F. Spiller, F. Inklaar, Liu Zongli, I. Wanders, M. Hiesgen, K. Anton, A. Barzewski, A. Juettner, M. Püttman, P.G. Niarchos, T. Szeifert, U. Kinkel, R. van Dijk, R. Kneer, B. Cunow, M. Niehues, R. Krenzin, M. Kruyswijk, M. Sevenster, M. Naumann and N. Vos. R. Faraggiana and G. Mathys gave unpublished information on spectral line widths for several programme stars. Unpublished information on $\lambda 5200$ photometry was obtained from H.M. Maitzen, H. Schneider, F.A. Catalano and W.W. Weiss. Information on older photometry was obtained through correspondence with E.H. Olsen, A.G. Davis Philip and P. Renson.

\section{REFERENCES}

Albrecht, R., Jenkner, H., Weiss, W.W., Wood, H.J. 1977, Astr. Ap. 58, 93.

Babcock, H.W. 1953, Pub. A.S.P. 65, 194.

Babcock, H.W. 1958, Ap. J. Suppl. 3, 141.

Bonsack, W.K., Pilachowski, C.A. 1974, Ap. J. 190, 327.

Borra, E.F., Landstreet, J.D. 1975, Publ. A.S.P. 87, 961.

Catalano, F.A., Renson, P. 1984, Astr. Ap. Suppl. 55, 371.

Deul, E.R., van Genderen, A.M. 1983, Astr. Ap. 118, 289.

Didelon, P. 1987, The Messenger 49, 5.

Dworetsky, M.M. 1982, Observatory 102, 138.

Gerth, E. 1990, in Hot Chemically Peculiar and Magnetic Stars, ed. G. Scholz Mitteilungen des Karl-Schwarzşchild-Observatoriums Tautenburg nr. 125, p.33. 
Hensberge, H., Manfroid, J., Schneider, H., Maitzen, H.M., Catalano, F.A., Renson, P., Weiss W.W., Floquet M. 1984, Astr. Ap. 132, 291.

Hensberge H., Manfroid, J., Sterken, C. 1993, in preparation (to be submitted to Astr. Ap. Suppl.)

Jones, T.J., Wolff, S.C. 1973 , Publ. A.S.P. 85, 760.

Kreidl, T.J. 1985, M.N.R.A.S. 216, 1017.

Kurtz, D.W. 1982, M.N.R.A.S. 200, 503.

Maitzen, H.M., Wood, H.J. 1977, Astr. Ap. 58, 389.

Manfroid, J., Mathys, G., Heck, A. 1985, Astr. Ap. 144, 251.

Manfroid, J., Sterken C. 1987, Astr. Ap. Suppl. 71, 539.

Manfroid, J., Sterken, C., Bruch, A., Burger, M., de Groot, M., Duerbeck, H.W., Duemmler, R., Figer, A., Hageman, T., Hensberge, H., Jorissen, A., Madejski, R., Mandel, H., Ott, H.-A., Reitermann A., Schulte-Ladbeck, R.E., Stahl, O., Steenman, H., vander Linden, D., Zickgraf, F.-J. 1991, Astr. Ap. Suppl. 87, 481.

Mathys, G. 1990, Astr. Ap. 232, 151.

Mathys, G. 1991, Astr. Ap. Suppl. 89, 121.

Mathys, G. 1992, in Proceedings I.A.U. Coll. 138 (this volume).

Musielok, B., Lange, D., Schöneich, W., Hildebrandt, G., Zelwanowa, E., Hempelmann, A.,Salmanov, G. 1980, Astron. Nachr. 301, 71.

Osawa, K., Nishimura, S., Ichimura J. 1965, Pub. A.S.J. 17, 199.

Preston, G.W. 1970, Ap. J. 160, 1059.

Preston, G.W. 1971, Ap. J. 164, 309.

Preston, G.W., Wolff S.C. 1970, Ap. J. 160, 1071.

Renson, P. 1977, IBVS nr. 1280.

Scholz, G. 1979, Astron. Nachr. 300, 213.

Schöneich, W. 1984, IBVS nr. 2569.

Sterken, C. 1983, The Messenger 33, 10.

van Genderen, A.M. 1971, Astr. Ap. 14, 48.

Winzer, J.E. 1974, Ph. D. Thesis, University of Toronto.

Wolff, S.C., Morrison, N.D. 1973, Publ. A.S.P. 85, 141.

Wolff, S.C. 1975, Ap. J. 202, 127.

Wood, H.J., Campusano, L.B. 1975, Astr. Ap. 45, 303. 